\title{
In vitro Fosfomycin Activity in Vancomycin-Resistant Enterococcus faecalis
}

\author{
Silvana Superti ${ }^{1}$, Cícero Armídio Gomes Dias ${ }^{2}$ and Pedro Alves d'Azevedo ${ }^{1,2}$ \\ ${ }^{1}$ Post-Graduation Program in Pathology of Health Sciences Federal University of Porto Alegre; ${ }^{2}$ Department of Microbiology and Parasitology \\ of Health Sciences Federal University of Porto Alegre; Porto Alegre, RS, Brazil
}

\begin{abstract}
Enterococci are part of the endogenous flora of human beings, are naturally resistant to several classes of antimicrobials, and are able to acquire resistance with relative ease. Currently the vancomycin-resistant enterococci are spread all over the world and treatment options for infections caused by it are often extremely limited. We assessed 193 vancomycin-resistant Enterococcus faecalis isolates collected from four different hospitals in Porto Alegre for their susceptibility to fosfomycin using the E-test and agar diffusion. Fosfomycin proved to be active in vitro against the great majority of isolates, indicating that it is a valid option in the treatment of these infections. Key-Words: Enterococcus faecalis, vancomycin-resistance, VRE, fosfomycin.
\end{abstract}

Enterococci are part of the normal intestinal human flora and can be involved in a great variety of infections, urinary tract infections being the most common [1]. Because they are intrinsically resistant to a wide range of antimicrobials, the choice of agents for treatment of infections is often very limited, and significant increases in resistance have been observed, as has been happening with the glycopeptides [2-4]. The first case of vancomycin-resistant enterococci (VRE) in Porto Alegre was reported in 2000, in an E. faecalis isolate that had the vanA gene [5]. More recently, this microorganism has been repeatedly isolated in several hospitals of the city, both in cultures from rectal swabs for investigation of carriers and from clinical materials associated with infectious processes. The multiresistance profile presented by these microorganisms makes it necessary to seek alternative treatments for these infections.

Fosfomycin is a natural antimicrobial of low molecular weight that penetrates the bacterial cell through two systems of permeases; one transports L- $\alpha$-glycerol-phosphate, and the another, which is inducible, takes D-glucose-6-phosphate into the interior of the bacterial cell. Once inside the cell, it acts by preventing cell wall synthesis, inhibiting by competition the enzyme UDP-N-acetylglucosamine-3-0enolpyruvate transferase. This action mechanism gives it a bactericidal effect, with a broad antimicrobial spectrum against both Gram-positive and Gram-negative bacteria [6,7].

The aim of this work was to assess in vitro fosfomycin activity in 193 vancomycin-resistant Enterococcus faecalis strains isolated in the city of Porto Alegre from 2003-2005.

\section{Material and Methods}

Isolates

One-hundred and ninety-three consecutive vancomycinresistant Enterococcus faecalis strains were tested. They were

Received on 4 August 2008; revised 7 December 2008.

Address for correspondence: Dr. Pedro A. d'Azevedo. Universidade Federal de Ciências da Saúde de Porto Alegre. Rua Sarmento Leite 245/ 204, Porto Alegre, RS, Brazil. Zip Code: 90050-170. Phone: 5551 33038740. Fax: 5551 32269756. E-mail: pedroaze@ufcspa.edu.br. Financial support: CNPq, UFCSPA.

The Brazilian Journal of Infectious Diseases 2009;13(2):123-124. (C) 2009 by The Brazilian Journal of Infectious Diseases and Contexto Publishing. All rights reserved. collected from patients hospitalized in four different health services: Complexo Irmandade Santa Casa de Porto Alegre, Complexo Nossa Senhora da Conceição, Hospital Ernesto Dornelles, and Hospital São Lucas, all of them located in the city of Porto Alegre, in southern Brazil. They were retrieved from a culture collection located in the Department of Microbiology, Universidade Federal de Ciências da Saúde de Porto Alegre. The isolates were kept at $-20^{\circ} \mathrm{C}$ in skim milk (Difco) plus 20\% glycerol. Enterococcus faecalis were identified by conventional biochemical tests [1] and vancomycin resistance was verified by MIC (Minimum Inhibitory Concentration) determination by the E-test (AB Biodisk, Sweden), according to the manufacturer's instructions, and by agar dilution according to the CLSI (2006).

Conventional Antimicrobial Susceptibility Testing Agar Diffusion Testing

This was performed according to the CLSI (2006), using disks containing $200 \mu \mathrm{g} / \mathrm{mL}$ of fosfomycin added to $50 \mu \mathrm{g} / \mathrm{mL}$ of D-glucose 6-phosphate (Cecon, São Paulo, Brazil).

\section{Gradient Diffusion Test (E-test)}

E-test strips (AB Biodisk, Sweden) were used, with fosfomycin gradient concentrations ranging from $0.04 \mu \mathrm{g} / \mathrm{mL}$ to $1,024 \mu \mathrm{g} / \mathrm{mL}$, added along with $50 \mu \mathrm{g} / \mathrm{mL}$ of glucose -6-phosphate. The tests were carried out as indicated by the manufacturer.

\section{Quality Control \\ Strain ATCC Enterococcus faecalis-29212 was used.}

\section{Results}

Only three isolates presented resistance by both methods, two with intermediate resistance (MIC of $128 \mu \mathrm{g} / \mathrm{mL}$ ) and one with full resistance (MIC above $256 \mu \mathrm{g} / \mathrm{mL}$, Table 1). The isolates presenting intermediate resistance were recovered from surveillance cultures for carriers, and the isolate presenting full resistance was from secretions from a surgical wound. The MICs ranged from 2 to $>256 \mu \mathrm{g} / \mathrm{mL}$, but they were mostly well below the breakpoint for resistance, with $\mathrm{MIC}_{50}$ equal to $8 \mu \mathrm{g} /$ $\mathrm{mL}$ and $\mathrm{MIC}_{90}$ equal to $16 \mu \mathrm{g} / \mathrm{mL}$. According to the CLSI (2006), isolates presenting MICs $\leq 64 \mu \mathrm{g} / \mathrm{mL}$ are considered susceptible. 
Table 1. Susceptibility of Enterococcus faecalis to fosfomycin by the E test.

\begin{tabular}{llllllllll}
\hline & & & \multicolumn{1}{c}{$\operatorname{MICs}(\mu \mathrm{g} / \mathbf{m L})$} & & & \\
\cline { 5 - 8 } & $\mathbf{2}$ & $\mathbf{4}$ & $\mathbf{8}$ & $\mathbf{1 6}$ & $\mathbf{3 2}$ & $\mathbf{6 4}$ & $\mathbf{1 2 8}$ & $\mathbf{2 5 6}$ & $\mathbf{2 5 6}$ \\
\hline Isolates $(\mathrm{N})$ & 3 & 6 & 121 & 41 & 19 & 02 & 0 & 1 \\
\hline
\end{tabular}

MICs = Minimum inhibitory concentrations.

\section{Discussion}

Several schemes have been proposed for treatment of VRE infections, but there are very few studies evaluating the activity of these in humans. New drugs have been developed, such as linezolid and quinupristine-dalfopristine, increasing the options for treatment of infections caused by this microorganism $[9,10]$. However, Enterococcus faecalis is intrinsically resistant to quinupristine-dalfopristine, and resistance against linezolid has already been reported [11]. Fosfomycin, owing to its broad antimicrobial spectrum, pharmacokinetics and administration routes, can be used in treating a diversity of infections such as bacteremias and urinary, respiratory, osteoarticular, and gynecological infections, among others [6,7]. Bacterial resistance to fosfomycin can occur by alterations in the transport system through the cell wall, by changes in the membrane, or rarely by enzymatic disruption of the drug [6,7]. Little is known about the in vitro activity of this antimicrobial against vancomycinresistant enterococci, and studies investigating its activity on vancomycin-resistant $E$. faecalis are scarce.

In a study by Allerberger et al. (1999), in which fosfomycin activity was tested against 118 samples of enterococci of different species, 100 being resistant to vancomycin, the $\mathrm{MIC}_{50}$ to fosfomycin was $32 \mu \mathrm{g} / \mathrm{mL}$ and the MIC $_{90}$ was $64 \mu \mathrm{g} / \mathrm{mL}$, with fosfomycin inhibiting more than $90 \%$ of the isolates. In another study by Perri et al. (2002), evaluating fosfomycin against 75 VRE clinical isolates, the reported in vitro susceptibility to fosfomycin was $100 \%$ among E. faecalis isolates and $67 \%$ among $E$. faecium isolates. In a more recent study performed by Abdulla \& Abdulla (2006), susceptibility to fosfomycin was found in $91 \%$ of vancomycin-resistant $E$. faecalis strains tested. These data are in agreement with the results obtained in our study, in which more than $98 \%$ of the isolates evaluated showed in vitro susceptibility to fosfomycin.

Shresta et al. (2000) reported success in the treatment of complicated urinary infection caused by this microorganism with the use of fosfomycin. Among the $193 \mathrm{E}$. faecalis isolates evaluated in our study, 39 (20.2\%) were recovered from the urine of hospitalized patients.

The results that we obtained with the two methods, agar diffusion and the E-test, were totally concordant, with 190 isolates categorized as susceptible to fosfomycin, two isolates as intermediate resistance, and only one as fully resistant, with MIC $>256 \mu \mathrm{g} / \mathrm{mL}$, according to the CLSI interpretive criteria (2006). While the treatment of multidrug-resistant enterococcal infection remains controversial and undefined, current therapies should be based on the local pattern of resistance. An analysis of the results obtained in our study showed that fosfomycin gives excellent in vitro activity against vancomycin-resistant $E$. faecalis isolated from hospitals of Porto Alegre in the 2003-2005 period, and could be a valid option in the treatment of infections caused by these multiresistant microorganisms, limiting the use of newer agents and possibly reducing the chance of development of further resistance.

\section{Acknowledgements}

The authors thank the staff of the Bacteriological Laboratories of the Hospitals for their help in collecting the Enterococcus spp. isolates. This study was supported in part by Universidade Federal de Ciências da Saúde de Porto Alegre and the Conselho Nacional de Desenvolvimento Científico e Tecnológico (CNPq).

\section{References}

1. Facklam R.R., Carvalho M.G., Teixeira L.M. History, Taxonomy, Biochemical Characteristics, and Antibiotic Susceptibility Testing of Enterococci. p1-54. In: The Enterococci, Pathogenesis, Molecular Biology and antibiotic Resistance. Gilmore M.S., Clewell D.B., Courvalin P., et al. (eds). $1^{\text {th }}$ ed., ASM Press, Washington, D.C, 2002.

2. Bonten M.J.M.,Willens R., Weistein R.A. Vancomycin-resistant enterococci: why are they here, and where do they come from. Lancet 2001;1:314-25.

3. Kak V., Chow J.W. Acquired Antibiotic Resistances in Enterococci. p. 355-384. In: The Enterococci, Pathogenesis, Molecular Biology and antibiotic Resistance. Gilmore M.S., Clewell D.B., Courvalin P., et al. (eds). $1^{\text {th }}$ ed, ASM Press, Washington, D.C, 2002.

4. Patel R. Clinical impact of vancomycin-resistant enterococci. J Antimicrob Chemother 2003;51:13-21.

5. d'Azevedo P.A., Kacman S.B., Schmalfuss T., et al. Primeiro caso de enterococo resistente à vancomicina isolado em Porto Alegre, RS. J Bras Patol Med Labor 2000;36(3):258.

6. Gobernado M. Fosfomicina. Rev Esp Quimioterap 2003;16:15-40.

7. Yao J.D.C., Moellering Jr. R.C. Antibacterial Agents. p. 10771113. In: Manual of Clinical Microbiology. Murray P.R., Baron E.J., Jorgensen J.H., et al. (eds). $9^{\text {th }}$ ed, ASM Press, Washington, D.C, 2007.

8. Clinical and Laboratory Standards Institute. Performance Standards for Antimicrobial Susceptibility Testing: Sixteenth Information Supplement M-100-516. 2006. CLSI, Wayne, PA, USA.

9. Kauffmann C.A. Therapeutic and preventative options for the management of vancomycin-resistant enterococcal infections. J Antimicrob Chemother 2003;51:23-30.

10. Noskin G.A. Tigecycline: a new glycylcycline for treatment of serious infections. Clin Infect Dis 2005;41:S303-14.

11. Johnson A.P., Tyssal L., Stockdale M.W., et al. Emerging LinezolidResistant Enterococcus faecalis and Enterococcus faecium isolated from two Austrian patients in the same intensive Care Unit. Eur J Clin Microbiol 2002; 21:751-4.

12. Allerberger F., Klare I. In vitro activity of fosfomycin against vancomycin-resistant enterococci. J Antimicrob Chemother 1999;43:211-7.

13. Perri M.B., Hershberger E., Ionescu M., et al. In vitro susceptibility of vancomycin resistant enterococci (VRE) to fosfomycin. Diag Microb Infect Dis 2002;42:269-71.

14. Abdulia F.E., Abdulia E.M. Antibiotic options for Enterococcus faecalis infections. Pak J Med Sci 2006;22(3):286-90.

15. Shresta N.K., Amuh D., Goldman M.P., et al. Treatment of a complicated vancomycin resistant enterococcal urinary tract infection with fosfomycin. Infect Dis Clin Prac 2000;9:368-71. 